\title{
Semi-Blind Adaptive Spatial Equalization for MIMO Systems with High-Order QAM Signalling
}

\author{
S. Chen, W. Yao, and L. Hanzo
}

\begin{abstract}
This contribution investigates semi-blind adaptive spatial filtering or equalisation for multiple-input multiple-output (MIMO) systems that employ high-throughput quadrature amplitude modulation (QAM) signalling. A minimum number of training symbols, equal to the number of receivers (we assume that the number of transmitters is no more than that of receivers), are first utilized to provide a rough least squares channel estimate of the system's MIMO channel matrix for the initialization of the spatial equalizers' weight vectors. A constant modulus algorithm aided soft decision-directed blind algorithm, originally derived for blind equalization of single-input single-output and single-input multiple-output systems employing high-order QAM signalling, is then extended to adapt the spatial equalizers for MIMO systems. This semi-blind scheme has a low computational complexity, and our simulation results demonstrate that it converges fast to the minimum mean-square-error spatial equalization solution.
\end{abstract}

Index Terms-Multiple-input multiple-output, quadrature amplitude modulation, spatial equalisation, semi-blind adaptive algorithm, concurrent constant modulus algorithm and soft decision-directed scheme.

\section{INTRODUCTION}

$\mathbf{M}$ ULTIPLE-INPUT multiple-output (MIMO) technologies are capable of substantially improving the achievable system's capacity and/or quality of service [1], [2], [3], [4]. However, the system's ability to approach the MIMO capacity heavily relies on the channel state information. Accurately estimating a MIMO channel is much more challenging than its single-input single-output (SISO) counterpart. The various MIMO channel estimation methods can be classified into three categories, namely, training-based methods, blind methods and semi-blind methods. Pure training-based schemes are computationally less demanding but a high proportion of training symbols is required in order to obtain a reliable MIMO channel estimate, which considerably reduces the achievable system throughput. The family of blind methods for joint channel estimation and data detection does not require training symbols and hence does not reduce the achievable system throughput, although this is achieved at the expense of high computational complexity. Moreover, blind joint channel estimation and data detection results in unavoidable estimation and decision ambiguities [5], [6]. Semi-blind schemes do not suffer from this ambiguity problem and are computationally simpler than their blind counterparts, at the cost of requiring a few training symbols.

Manuscript received August 1, 2007; revised October 31, 2007; accepted December 17, 2007. The associate editor coordinating the review of this letter and approving it for publication was G. Vitetta.

The authors are with School of Electronics and Computer Science, University of Southampton, Southampton SO17 1BJ, UK (e-mail: \{sqc, wy07r, 1h\}@ecs.soton.ac.uk).

Digital Object Identifier 10.1109/T-WC.2008.070852
Many semi-blind methods have been developed for MIMO systems. In the schemes of [7], [8], [9], [10], a few training symbols are used to provide an initial MIMO channel estimate, and the channel estimator as well as the data detector iteratively exchange their information, where the channel estimator relies on decision-directed adaptation. In [11], the MIMO channel matrix is decomposed into the product of a whitening matrix and a rotational unitary matrix. The first matrix is estimated blindly while the second is estimated with the aid of training symbols. In contrast to these proposals, recently we have proposed a novel semi-blind scheme for joint maximum likelihood (ML) channel estimation and data detection [12], where the joint ML channel and data estimation optimization process is decomposed into two levels. At the upper level a global optimization algorithm searches for an optimal channel estimate, while at the lower level a ML data detector recovers the transmitted data. Joint ML channel estimation and data detection is achieved by iteratively exchanging information between the channel estimator and the data detector. Assume that the MIMO system has $n_{T}$ transmitters and $n_{R}$ receivers. A minimum number of training symbols, equal to $\max \left\{n_{R}, n_{T}\right\}$, are used to provide an initial least squares channel estimate (LSCE) [13] for aiding the upper level channel estimator to improve convergence. The employment of a minimum training overhead has an additional benefit in terms of avoiding the ambiguities inherent in pure blind joint channel estimation and data detection.

Semi-blind joint ML schemes are attractive because they are capable of approaching the optimal joint ML solution. However, for MIMO systems that employ high-throughput quadrature amplitude modulation (QAM) signalling [14], these schemes become computationally prohibitive owe to the high complexity of ML data detection. Instead of performing joint channel estimation and data detection, we consider direct spatial filtering or equalization for MIMO systems that employ high-order QAM schemes. In particular, we consider the lowcomplexity minimum mean square error (MMSE) solution as the optimal design for the spatial equalization. Thus, we assume that for the MIMO system concerned the number of transmitters is no more than that of receivers. The proposed method is semi-blind as we employ a minimum number of pilots, which is equal to the number of receivers, to estimate the MIMO channel matrix via the LSCE. The resulting LSCE is used to initialize the weight vectors of the spatial equalizers. In general, this initialization is not sufficiently accurate to achieve an "opening-eye" and, therefore, it is unsafe to carry out decision-directed (DD) adaptation for the spatial equalizers. We propose to use a constant modulus algorithm (CMA) assisted soft DD (SDD) blind adaptive algorithm to adapt the 
spatial equalizers. The concurrent CMA and SDD algorithm was originally derived for blind equalization of single-input single-output (SISO) QAM systems [15], and it was extended to single-input multiple-output (SIMO) systems in [16]. This blind adaptive scheme has a very low computational complexity. In the present MIMO application, owing to the initial information provided by the training pilots, the algorithm converges much faster than the pure blind adaptation case, and it is capable of approaching the performance of the MMSE spatial equalizers based on the perfect channel knowledge, as will be shown in our simulation study.

To the best of our knowledge, this is the first time that a very low-complexity stochastic gradient adaptive semi-blind spatial equalization scheme is proposed for MIMO-aided highorder QAM schemes. We have performed extensive literature survey, and we have only found one journal paper accepted for publication [17]. In [17], the authors propose to adapt the spatial equalizer by minimizing the combined cost function of the training-based sum of the squared errors and a higherorder statistic (HOS) aided criterion using a block-data based gradient algorithm. In terms of computational requirements, the complexity of the block-data based algorithm in [17] is significantly higher than that of our proposed stochastic gradient algorithm. In terms of the achievable equalization performance, our simpler stochastic gradient scheme actually outperforms the more complex block-data based gradient scheme of [17]. This is because the blind adaptive process in the semi-blind scheme of [17] is based on the HOS (e.g. CMA) criterion, while our blind adaptive process is based on the HOS (CMA) aided SDD criterion. The latter can approach the optimal MMSE solution more accurately and achieve a faster convergence, as a benefit of the fact that SDD adaptation is more like the true training.

Throughout this contribution we adopt the following notational conventions. Boldface capitals and lower-case letters stand for matrices and vectors, respectively, while $\mathbf{I}_{K}$ denotes the $K \times K$ identity matrix. Furthermore, $(\bullet)^{T}$ and $(\bullet)^{H}$ are the transpose and Hermitian operators, respectively, while $\|\bullet\|$ and $|\bullet|$ denote the norm and magnitude operators, respectively. $E[\bullet]$ is the expectation operator, while $(\bullet)^{*}$ denotes the complex conjugate. Finally, $j=\sqrt{-1}$.

\section{SySTEM MOdeL}

We consider a MIMO system consisting of $n_{T}$ transmitters and $n_{R}$ receivers, which communicates over flat fading channels. The system is described by the following well-known MIMO model

$$
\mathbf{x}(k)=\mathbf{H} \mathbf{s}(k)+\mathbf{n}(k),
$$

where $k$ is the symbol index, $\mathbf{H}$ denotes the $n_{R} \times n_{T}$ narrowband MIMO channel matrix, $\mathbf{s}(k)=\left[s_{1}(k) s_{2}(k) \cdots s_{n_{T}}(k)\right]^{T}$ is the transmitted symbols vector of the $n_{T}$ transmitters with the symbol energy given by $E\left[\left|s_{m}(k)\right|^{2}\right]=\sigma_{s}^{2}$ for $1 \leq m \leq n_{T}, \mathbf{x}(k)=\left[x_{1}(k) x_{2}(k) \cdots x_{n_{R}}(k)\right]^{T}$ denotes the received signal vector, and $\mathbf{n}(k)=\left[n_{1}(k) n_{2}(k) \cdots n_{n_{R}}(k)\right]^{T}$ is the complex-valued Gaussian white noise vector associated with the MIMO channels with $E\left[\mathbf{n}(k) \mathbf{n}^{H}(k)\right]=2 \sigma_{n}^{2} \mathbf{I}_{n_{R}}$. We assume that $n_{T} \leq n_{R}$ and the channels are non-dispersive. Frequency selective channels can be made narrowband using for example the orthogonal frequency division multiplexing technique [18]. Alternatively, a common detection strategy for frequency-selective MIMO channels is first to achieve temporal equalization and then to perform spatial equalization [19]. Temporal equalization can be accomplished conveniently using blind equalisation methods based on second-order statistics (SOS) [20], [21]. These SOS-based blind equalization schemes achieve temporal equalization while leaving the ambiguity of an instantaneous mixture [6], which is equivalent to a narrowband MIMO system.

Specifically, the narrow-band MIMO channel matrix is defined by $\mathbf{H}=\left[h_{l, m}\right]$, for $1 \leq l \leq n_{R}$ and $1 \leq m \leq n_{T}$, where $h_{l, m}$ denotes the non-dispersive channel coefficient linking the $m$-th transmitter to the $l$-th receiver. Furthermore, the fading is assumed to be sufficiently slow, so that during the time period of a transmission block or frame, all the entries in the MIMO channel matrix $\mathbf{H}$ may be deemed unchanged. From frame to frame, the channel impulse response taps $h_{l, m}$ are independently and identically distributed (i.i.d.) complexvalued Gaussian processes with zero mean and $E\left[\left|h_{l, m}\right|^{2}\right]=$ 1. The modulation scheme is the $M$-QAM and, therefore, the transmitted data symbols $s_{m}(k), 1 \leq m \leq n_{T}$, take the values from the $M$-QAM symbol set defined by

$$
\mathcal{S} \triangleq\left\{s_{i, q}=u_{i}+j u_{q}, 1 \leq i, q \leq \sqrt{M}\right\}
$$

with the real-part symbol $\Re\left[s_{i, q}\right]=u_{i}=2 i-\sqrt{M}-1$ and the imaginary-part symbol $\Im\left[s_{i, q}\right]=u_{q}=2 q-\sqrt{M}-1$. The average signal-to-noise ratio (SNR) of the system is defined as

$$
\mathrm{SNR}=n_{T} \times \sigma_{s}^{2} / 2 \sigma_{n}^{2} .
$$

A bank of the spatial filters or equalizers

$$
y_{m}(k)=\mathbf{w}_{m}^{H} \mathbf{x}(k), 1 \leq m \leq n_{T},
$$

are used to detect the transmitted symbols $s_{m}(k)$ for $1 \leq$ $m \leq n_{T}$, where $\mathbf{w}_{m}$ is the $n_{R} \times 1$ complex-valued weight vector of the $m$-th spatial equalizer. With the perfect channel knowledge, the optimal MMSE solutions for the $n_{T}$ spatial equalizers are given by

$$
\mathbf{w}_{\mathrm{MMSE}, m}=\left(\mathbf{H H}^{H}+\frac{2 \sigma_{n}^{2}}{\sigma_{s}^{2}} \mathbf{I}_{n_{R}}\right)^{-1} \mathbf{h}_{m}, 1 \leq m \leq n_{T},
$$

where $\mathbf{h}_{m}$ denotes the $m$-th column of the channel matrix $\mathbf{H}$.

\section{The Proposed Semi-Blind Algorithm}

Let the number of training symbols be $K$, and denote the available training data as $\mathbf{X}_{K}=[\mathbf{x}(1) \mathbf{x}(2) \cdots \mathbf{x}(K)]$ and $\mathbf{S}_{K}=[\mathbf{s}(1) \mathbf{s}(2) \cdots \mathbf{s}(K)]$. The LSCE of the MIMO channel matrix $\mathbf{H}$ based on $\left\{\mathbf{S}_{K}, \mathbf{X}_{K}\right\}$ is readily given as

$$
\hat{\mathbf{H}}=\mathbf{X}_{K} \mathbf{S}_{K}^{H}\left(\mathbf{S}_{K} \mathbf{S}_{K}^{H}\right)^{-1} .
$$

As a byproduct of the LSCE (6), an estimated noise variance is also produced as

$$
2 \hat{\sigma}_{n}^{2}=\frac{1}{K \cdot n_{R}}\left\|\mathbf{X}_{K}-\hat{\mathbf{H}} \mathbf{S}_{K}\right\|^{2} .
$$

In order to maintain throughput, the number of training pilots should be as small as possible. To ensure that $\mathbf{S}_{K} \mathbf{S}_{K}^{H}$ has a full rank, we will choose $K=\max \left\{n_{T}, n_{R}\right\}=n_{R}$. That 
is, we assume that $K=n_{R}$ is the minimum number of training symbols. The rough LSCE $\hat{\mathbf{H}}$ is utilized to provide the initialization of the spatial equalizers' weight vectors via the MMSE solutions

$$
\mathbf{w}_{m}(0)=\left(\hat{\mathbf{H}} \hat{\mathbf{H}}^{H}+\frac{2 \hat{\sigma}_{n}^{2}}{\sigma_{s}^{2}} \mathbf{I}_{n_{R}}\right)^{-1} \hat{\mathbf{h}}_{m}, 1 \leq m \leq n_{T},
$$

where $\hat{\mathbf{h}}_{m}$ denotes the $m$-th column of $\hat{\mathbf{H}}$. Because the training data are insufficient, the weight vectors (8) are not sufficiently accurate to open the eye. Therefore, DD adaptation is generally unsafe. However, we can apply the concurrent CMA and SDD blind scheme [15], [16] to adapt the spatial filters (4) with $\mathbf{w}_{m}(0)$ of (8) as their initial weight vectors. Let the weight vector of the $m$-th spatial equalizer be split into two parts, yielding $\mathbf{w}_{m}=\mathbf{w}_{m, c}+\mathbf{w}_{m, d}$, and denote the spatial equalizer's output at sample $k$ as $y_{m}(k)=\mathbf{w}_{m}^{H}(k) \mathbf{x}(k)$. The initial $\mathbf{w}_{m, c}$ and $\mathbf{w}_{m, d}$ can simply be set to $\mathbf{w}_{m, c}(0)=$ $\mathbf{w}_{m, d}(0)=0.5 \mathbf{w}_{m}(0)$, with $\mathbf{w}_{m}(0)$ given in (8).

Specifically the weight vector $\mathbf{w}_{m, c}$ is updated using the classical CMA [22], [23]

$$
\left.\begin{array}{l}
\varepsilon_{m}(k)=y_{m}(k)\left(\Delta-\left|y_{m}(k)\right|^{2}\right), \\
\mathbf{w}_{m, c}(k+1)=\mathbf{w}_{m, c}(k)+\mu_{\mathrm{CMA}} \varepsilon_{m}^{*}(k) \mathbf{x}(k),
\end{array}\right\}
$$

where $\Delta=E\left[\left|s_{i}(k)\right|^{4}\right] / E\left[\left|s_{i}(k)\right|^{2}\right]$ and $\mu_{\mathrm{CMA}}$ is the step size of the CMA. The weight vector $\mathbf{w}_{m, d}$ by contrast is updated using the SDD scheme [15], [16], which has its root in the blind equalisation scheme of [24]. The complex phasor plane is divided into the $M / 4$ rectangular regions, and each region $\mathcal{S}_{i, l}$ contains four symbol points as defined in the following

$$
\mathcal{S}_{i, l}=\left\{s_{p, q}, p=2 i-1,2 i, q=2 l-1,2 l\right\},
$$

where $1 \leq i, l \leq \sqrt{M} / 2$. If the spatial equalizer's output $y_{m}(k) \in \mathcal{S}_{i, l}$, a local approximation of the marginal probability density function (PDF) of $y_{m}(k)$ is given by [15], [16]

$$
\hat{p}\left(\mathbf{w}_{m}, y_{m}(k)\right) \approx \sum_{p=2 i-1}^{2 i} \sum_{q=2 l-1}^{2 l} \frac{1}{8 \pi \rho} e^{-\frac{\left|y_{m}(k)-s_{p, q}\right|^{2}}{2 \rho}},
$$

where $\rho$ defines the cluster width associated with the four clusters of each region $\mathcal{S}_{i, l}$. The SDD algorithm is designed to maximize the log of the local marginal PDF criterion $E\left[J_{\mathrm{LMAP}}\left(\mathbf{w}_{m}, y_{m}(k)\right)\right]$, where $J_{\mathrm{LMAP}}\left(\mathbf{w}_{m}, y_{m}(k)\right)=$ $\rho \log \left(\hat{p}\left(\mathbf{w}_{m}, y_{m}(k)\right)\right)$, via a stochastic gradient optimization. Specifically, $\mathbf{w}_{m, d}$ is updated according to [15], [16]

$$
\mathbf{w}_{m, d}(k+1)=\mathbf{w}_{m, d}(k)+\mu_{\mathrm{SDD}} \frac{\partial J_{\mathrm{LMAP}}\left(\mathbf{w}_{m}(k), y_{m}(k)\right)}{\partial \mathbf{w}_{m, d}},
$$

where $\mu_{\mathrm{SDD}}$ is the step size of the SDD, and

$$
\begin{gathered}
\frac{\partial J_{\mathrm{LMAP}}\left(\mathbf{w}_{m}, y_{m}(k)\right)}{\partial \mathbf{w}_{m, d}}= \\
\frac{1}{Z_{N}} \sum_{p=2 i-1}^{2 i} \sum_{q=2 l-1}^{2 l} e^{-\frac{\left|y m_{m}(k)-s_{p, q}\right|^{2}}{2 \rho}}\left(s_{p, q}-y_{m}(k)\right)^{*} \mathbf{x}(k),
\end{gathered}
$$

with the normalization factor

$$
Z_{N}=\sum_{p=2 i-1}^{2 i} \sum_{q=2 l-1}^{2 l} e^{-\frac{\left|y_{m}(k)-s_{p, q}\right|^{2}}{2 \rho}} .
$$

TABLE I

THE Simulated STATIONARY $4 \times 4$ 64-QAM MIMO SYSTEM

\begin{tabular}{|rrrr|}
\hline$-1.4-0.6 j$ & $0.5+1.1 j$ & $0.4-0.8 j$ & $-0.6-0.3 j$ \\
$1.7-0.3 j$ & $1.3-0.3 j$ & $-0.1-1.4 j$ & $-0.6-0.5 j$ \\
$1.0+0.5 j$ & $-0.6+0.8 j$ & $-0.6-0.2 j$ & $-0.3+0.2 j$ \\
$1.2-1.3 j$ & $-0.7+1.0 j$ & $0.9-0.3 j$ & $-0.1+0.7 j$ \\
\hline
\end{tabular}

The choice of the cluster width $\rho$, defined in the context of the local PDF (11), should ensure a proper separation of the four clusters of $\mathcal{S}_{i, l}$. As the minimum distance between the two neighboring constellation points is $2, \rho$ is typically chosen to be less than 1 . If the value of $\rho$ is too large, a desired degree of separation may not be achieved. On the other hand, if too small a $\rho$ value is used, the algorithm attempts to impose an overly tight control on the size of clusters and hence may fail to achieve its goal. Apart from these two extreme situations, the performance of the algorithm is not overly sensitive to the value of $\rho$ employed and an appropriate $\rho$ can easily be chosen from a large range of values. More specifically, when the equalization objective is accomplished, $y_{m}(k) \approx s_{m}(k)+e_{m}(k)$, where $e_{m}(k)$ is Gaussian distributed with zero mean. Therefore, the value of $\rho$ is related to the variance of $e_{m}(k)$, which is $2 \sigma_{n}^{2} \mathbf{w}_{m}^{H} \mathbf{w}_{m}$. Thus, for high SNR situations, small $\rho$ is desired, while for low SNR cases, large $\rho$ is preferred. Because of the information provided by the training pilots in the form of the initial weight vectors (8), smaller $\rho$ can be used, compared with the case of pure blind adaptation in [15], [16], which leads to better steady-state performance. Soft decision nature becomes explicit in (13), because rather than committing to a single hard decision $\mathcal{Q}\left[y_{m}(k)\right]$, where $\mathcal{Q}[\bullet]$ denotes the quantization operator, as the hard DD scheme would, alternative decisions are also considered in the local region $\mathcal{S}_{i, l}$ that includes $\mathcal{Q}\left[y_{m}(k)\right]$, and each tentative decision is weighted by an exponential term $e^{\{\bullet\}}$, which is a function of the distance between the equalizer's soft output $y_{m}(k)$ and the tentative decision $s_{p, q}$. This soft decision nature substantially reduces the risk of error propagation and achieves faster convergence, compared with the hard DD scheme [15], [16].

\section{Simulation Study}

A simulation study was carried out to investigate the proposed semi-blind spatial equalization scheme based on the concurrent CMA and SDD algorithm. The achievable performance was assessed in the simulation using the symbol error rate (SER). The analytical SER for the spatial equalizer (4) with the weight vector $\mathbf{w}_{m}$ is given in the Appendix.

Stationary channel realization. We considered a MIMO system with $n_{T}=4$ and $n_{R}=4$, and the modulation scheme was 64-QAM. The simulated stationary $4 \times 4$ MIMO channel matrix $\mathbf{H}$ is listed in Table I. The number of pilot symbols used for the semi-blind scheme was $K=4$. Firstly, trainingbased spatial filtering was demonstrated. Given $K$ training symbols, the LSCE $\hat{\mathbf{H}}$ was obtained, which was then used to calculate the MMSE solution for the weight vectors of the four spatial equalizers. The average SER performance over all the four spatial equalizers as a function of the training symbols $K$ are depicted in Fig. 1, with the average SER of the true MMSE spatial equalizers calculated based on the true MIMO 


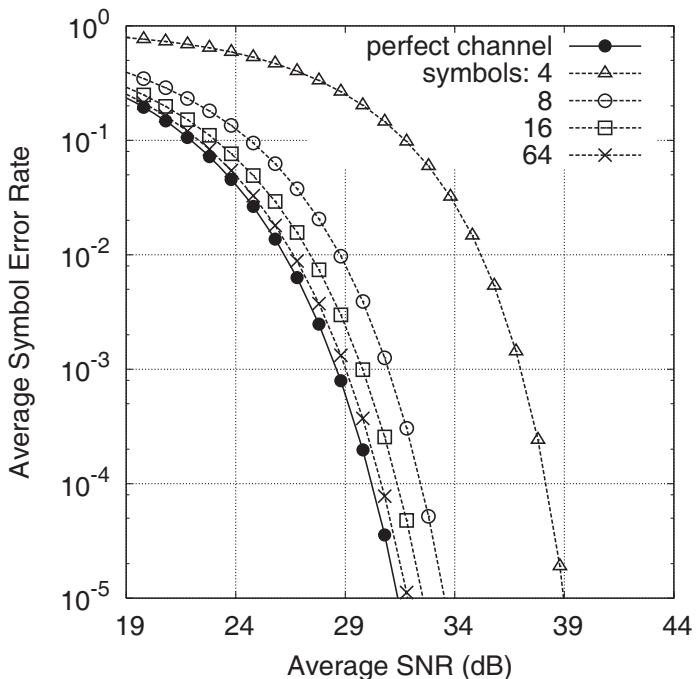

Fig. 1. Average symbol error rate performance of the training-based spatial equalization given different numbers of training symbols, in comparison with the case of perfect channel knowledge, for the stationary $4 \times 4$ 64-QAM MIMO system.

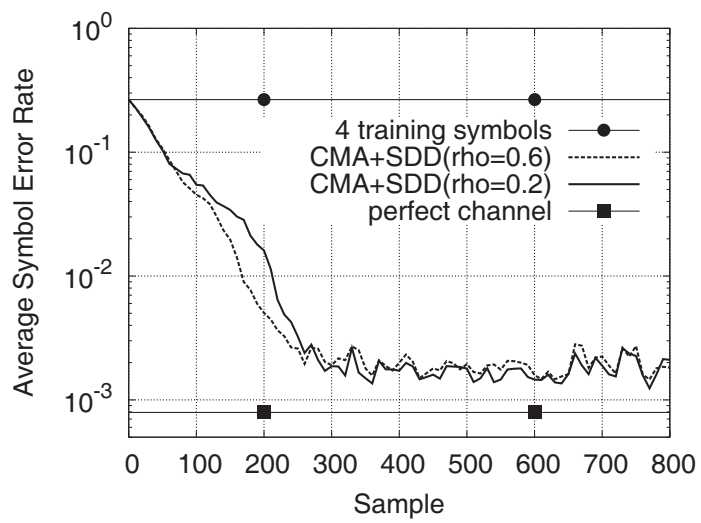

Fig. 2. Learning curves of the concurrent CMA and SDD scheme in terms of the SER average over all the four spatial equalizers and over ten different runs for the stationary $4 \times 464$-QAM MIMO system, given the average SNR of $28.8 \mathrm{~dB}, \mu_{\mathrm{CMA}}=4 \times 10^{-7}, \mu_{\mathrm{SDD}}=2 \times 10^{-4}$ and two values of cluster width $\rho$.

channel matrix $\mathbf{H}$ as the benchmark. It can be seen from Fig. 1 that the training-based scheme required more than 64 training pilots to closely approach the optimal MMSE performance. For this simulated MIMO system, the 4-th spatial equalizer had the worst SER performance while the 1st spatial equalizer had the best SER performance. Therefore, the average SER performance shown in Fig. 1 was dominated by the worst case of the 4-th spatial equalizer.

The proposed semi-blind spatial equalization scheme was next investigated. Given the SNR of $28.8 \mathrm{~dB}, K=4$ training pilots were first used to provide the initial weight vectors of the four spatial equalizers according to (8). The appropriate values for the step size of the CMA as well as the step size of the SDD were found empirically, and they were chosen to be $\mu_{\mathrm{CMA}}=4 \times 10^{-7}$ and $\mu_{\mathrm{SDD}}=2 \times 10^{-4}$, respectively. A wide range of values were found to be suitable for the cluster width of the SDD. Fig. 2 plots the learning curves of the combined CMA and SDD adaptive algorithm in terms of the average SER over all the four spatial equalizers and over ten

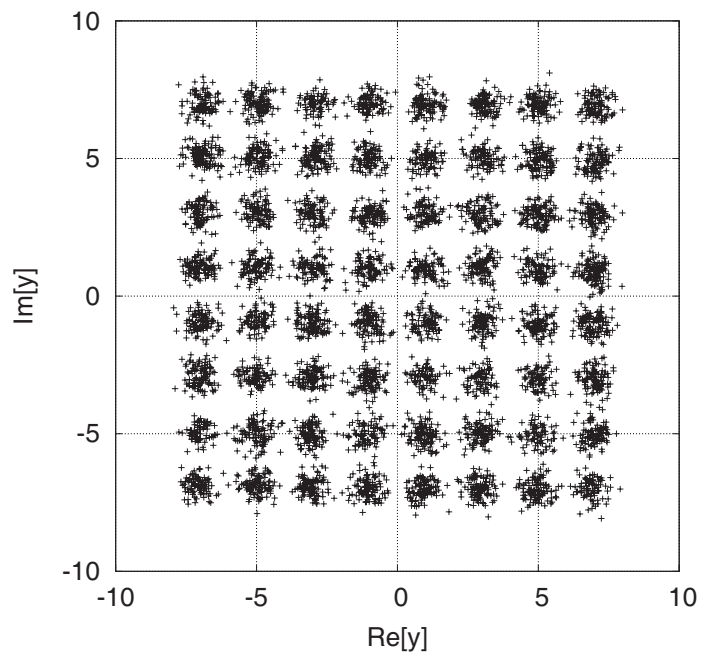

Fig. 3. The 4-th spatial equalizer's output constellation after blind adaptation given average SNR of $28.8 \mathrm{~dB}$ for the stationary $4 \times 4$ 64-QAM MIMO system, shown with 6000 data points.

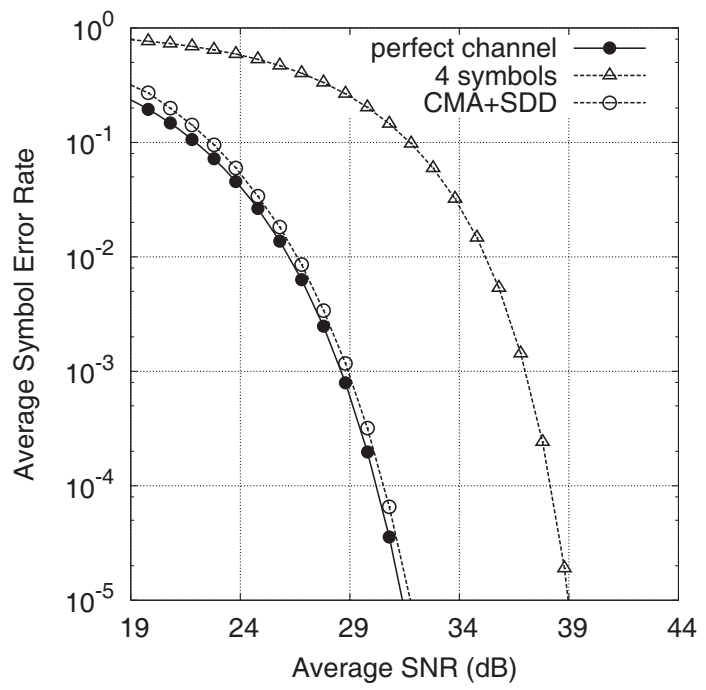

Fig. 4. Average symbol error rate performance of the proposed semi-blind spatial equalization scheme with four training symbols, in comparison with the cases of training only based on four training symbols and perfect channel knowledge, for the stationary $4 \times 464-\mathrm{QAM}$ MIMO system.

different runs. More specifically, two learning curves obtained with $\rho=0.2$ and $\rho=0.6$, respectively, are shown in Fig. 2, where it can be observed that the case of $\rho=0.2$ yielded a lightly better steady-state SER performance while the case of $\rho=0.6$ produced a marginally faster convergence. It can be concluded from Fig. 2 that $\rho$ in the range of $[0.2,0.6]$ were appropriate. It is also observed from Fig. 2 that, aiding by the information provided by the four training pilots, the convergence rate of the concurrent CMA and SDD algorithm was much faster than the pure blind adaptive counterpart of [15], [16]. Furthermore, the proposed semi-blind scheme is capable of approaching the optimal MMSE solution, as can be seen in Fig. 2.

Given the average SNR of $28.8 \mathrm{~dB}, K=4$ training symbols were insufficient for the 4-th spatial equalizer. The eye diagram of the equalizer's output constellation for the 4th spatial equalizer before the blind adaptation, i.e. with the 


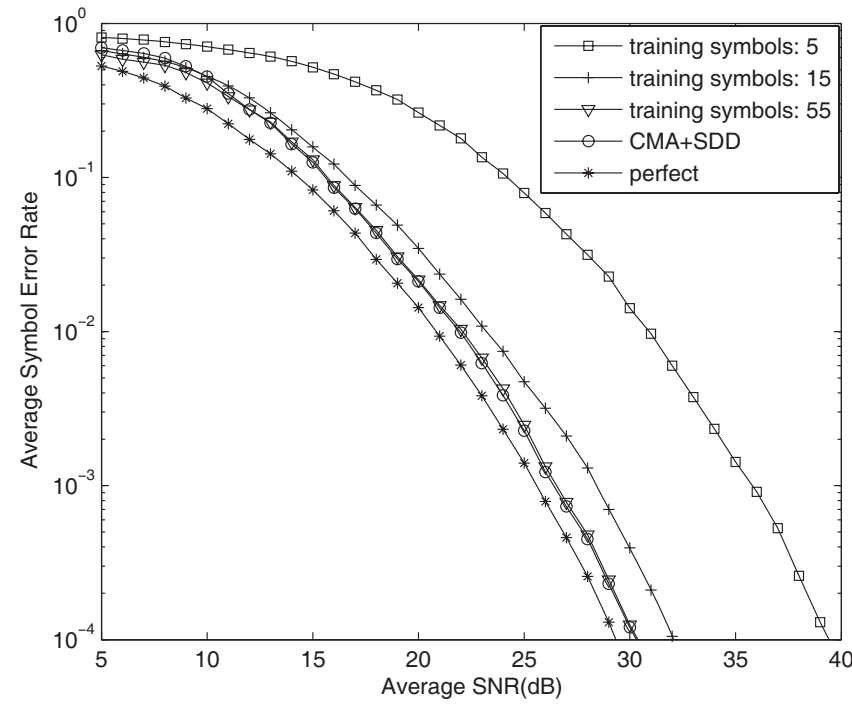

Fig. 5. Average symbol error rate performance of the proposed semi-blind spatial equalization scheme with five training symbols, in comparison with the cases of training only based on different numbers of training symbols and perfect channel knowledge, averaged over 100 realizations of the flat Rayleigh fading $5 \times 4$ 16-QAM MIMO system.

weight vector $\mathbf{w}_{4}(0)$, was completely closed. By contrast, the 4-th spatial equalizer's output constellation after blind adaptation is illustrated in Fig. 3, clearly showing that the eye was opened. Finally, the average SER performance achieved by the proposed semi-blind spatial equalization scheme with assistant of four training pilots is compared with that of the perfect channel knowledge as well as that of the training-based scheme utilizing only four training pilots. The results showing in Fig. 4 clearly confirm that the proposed semi-blind spatial equalization scheme closely approached the optimal MMSE spatial equalization solution. For the purely training based scheme to achieve a similar performance, at least 64 training symbols are required, as can be observed in Fig. 1.

Flat Rayleigh fading channel. We considered a MIMO system with $n_{T}=4, n_{R}=5$ and the 16-QAM modulation scheme. The simulated channel impulse response taps $h_{l, m}$, $1 \leq l \leq 5$ and $1 \leq m \leq 4$, were i.i.d. complex-valued Gaussian processes with zero mean and $E\left[\left|h_{l, m}\right|^{2}\right]=1$, and the performance was averaged over 100 channel realizations. The number of pilot symbols used for the semi-blind scheme was $K=5$. The average SER performance over all the four spatial equalizers for the purely training based scheme with 5,15 and 55 training symbols, respectively, as well as the proposed semi-blind spatial equalization scheme with aid of 5 training symbols are shown in Fig. 5, in comparison with the achievable performance given the perfect channel knowledge. The step size of the CMA as well as the step size and cluster width of the SDD were empirically set to $\mu_{\mathrm{CMA}}=2 \times 10^{-6}$, $\mu_{\mathrm{SDD}}=5 \times 10^{-4}$ and $\rho=0.5$. The blind adaptive process was observed to achieve convergence typically within 300 samples. It can be seen from Fig. 5 that to achieve a similar performance as the semi-blind CMA-SDD scheme the training based scheme required 55 training symbols.

\section{CONCLUSIONS}

A semi-blind spatial equalization scheme has been proposed for narrow-band MIMO systems that employ high throughput QAM signalling. A minimum number of training symbols, equal to the number of receivers, is used to estimate the MIMO channel matrix and the resulting rough LSCE is utilized for the initialization of the spatial equalizers. The CMA aided SDD blind adaptive scheme is then adopted to adapt the spatial equalizers. The proposed semi-blind spatial equalization scheme has a very low computational complexity, and our simulation study has confirmed that this semi-blind concurrent CMA and SDD algorithm converges much faster than its pure blind counterpart. Simulation results have also shown that the proposed semi-blind spatial equalization scheme is capable of approaching the optimal MMSE spatial equalization solution calculated based on the perfect channel knowledge.

\section{APPENDIX}

The analytical SER for the spatial equalizer (4) with the weight vector $\mathbf{w}_{m}$ can be derived, similar to the case of adaptive beamforming [25]. Define the combined response of the $m$-th spatial filter and the MIMO channel as $\mathbf{w}_{m}^{H} \mathbf{H}=$ $\left[c_{m, 1} c_{m, 2} \cdots c_{m, n_{T}}\right]$, and assume that $c_{m, m}=c_{R_{m, m}}+j c_{I_{m, m}}$ satisfies $c_{R_{m, m}}>0$ and $c_{I_{m, m}}=0$. The MMSE solution for the spatial filter, calculated based on the true MIMO channel matrix, meets this condition. For our proposed semiblind spatial filter, this condition is generally met, as the filter approaches the MMSE solution. If this condition is not satisfied, a rotation operation can always be performed on the weight vector to guarantee this condition [25]. The SER of the spatial equalizer $y_{m}(k)$ is given by

$$
\begin{aligned}
P_{E}\left(\mathbf{w}_{m}\right)= & P_{E_{R}}\left(\mathbf{w}_{m}\right)+P_{E_{I}}\left(\mathbf{w}_{m}\right) \\
& -P_{E_{R}}\left(\mathbf{w}_{m}\right) P_{E_{I}}\left(\mathbf{w}_{m}\right),
\end{aligned}
$$

where $P_{E_{R}}\left(\mathbf{w}_{m}\right)$ and $P_{E_{I}}\left(\mathbf{w}_{m}\right)$ are the real-part and imaginary-part SERs, respectively. Note $\mathbf{x}(k)=\overline{\mathbf{x}}(k)+\mathbf{n}(k)$. Similarly, $y_{m}(k)=\bar{y}_{m}(k)+e_{m}(k)$, where $e_{m}(k)$ is Gaussian distributed with zero mean and $E\left[\left|e_{m}(k)\right|^{2}\right]=2 \sigma_{n}^{2} \mathbf{w}_{m}^{H} \mathbf{w}_{m}$. The noise-free part $\bar{y}_{m}(k)$ takes values from the scalar set $\mathcal{Y}_{m}$ that contains $N_{s}=M^{n_{T}}$ points. The set $\mathcal{Y}_{m}$ can be divided into the $M$ subsets conditioned on $s_{m}(k)$ as

$$
\mathcal{Y}_{m}^{(l, q)} \triangleq\left\{\bar{y}_{m, i}^{(l, q)} \in \mathcal{Y}_{m}, 1 \leq i \leq N_{s b}: s_{m}(k)=s_{l, q}\right\},
$$

for $1 \leq l, q \leq \sqrt{M}$, where the size of $\mathcal{Y}_{m}^{(l, q)}$ is $N_{s b}=N_{s} / M$. The subset $\mathcal{Y}_{m}^{(l, q)}$ is completely specified by the MIMO channel matrix $\mathbf{H}$. The SER $P_{E}\left(\mathbf{w}_{m}\right)$ can be calculated based on a single subset $\mathcal{Y}_{m}^{(l, q)}$ [25]. [25]

Expressing $\bar{y}_{m, i}^{(l, q)}=\bar{y}_{R_{m, i}}^{(l, q)}+j \bar{y}_{I_{m, i}}^{(l, q)}$, it can be shown that

$$
P_{E_{R}}\left(\mathbf{w}_{m}\right)=\gamma \frac{1}{N_{s b}} \sum_{i=1}^{N_{s b}} Q\left(g_{R_{m, i}}^{(l, q)}\left(\mathbf{w}_{m}\right)\right)
$$

and

$$
P_{E_{I}}\left(\mathbf{w}_{m}\right)=\gamma \frac{1}{N_{s b}} \sum_{i=1}^{N_{s b}} Q\left(g_{I_{m, i}}^{(l, q)}\left(\mathbf{w}_{m}\right)\right)
$$


where $\gamma=\frac{2 \sqrt{M}-2}{\sqrt{M}}$,

$$
\begin{aligned}
Q(u) & =\frac{1}{\sqrt{2 \pi}} \int_{u}^{\infty} e^{-\frac{z^{2}}{2}} d z, \\
g_{R_{m, i}}^{(l, q)}\left(\mathbf{w}_{m}\right) & =\frac{\bar{y}_{R_{m, i}}^{(l, q)}-c_{R_{m, m}}\left(u_{l}-1\right)}{\sigma_{n} \sqrt{\mathbf{w}_{m}^{H} \mathbf{w}_{m}}}, \\
g_{I_{m, i}}^{(l, q)}\left(\mathbf{w}_{m}\right) & =\frac{\bar{y}_{I_{m, i}}^{(l, q)}-c_{R_{m, m}}\left(u_{q}-1\right)}{\sigma_{n} \sqrt{\mathbf{w}_{m}^{H} \mathbf{w}_{m}}} .
\end{aligned}
$$

Note that the SER is invariant to a positive scaling of $\mathbf{w}_{m}$.

\section{REFERENCES}

[1] G. J. Foschini and M. J. Gans, "On limits of wireless communications in a fading environment when using multiple antennas," Wireless Personal Commun., vol. 6, no. 3, pp. 311-335, 1998.

[2] I. E. Telatar, "Capacity of multi-antenna Gaussian channels," European Trans. Telecommun., vol. 10, no. 6, pp. 585-595, 1999.

[3] T. L. Marzetta and B. M. Hochwald, "Capacity of a mobile multipleantenna communication link in Rayleigh flat fading," IEEE Trans. Inform. Theory, vol. 45, no. 1, pp. 139-157, 1999.

[4] A. J. Paulraj, D. A. Gore, R. U. Nabar, and H. Bölcskei, "An overview of MIMO communications-a key to gigabit wireless," Proc. IEEE, vol. 92, no. 2, pp. 198-218, 2004.

[5] L. Tang, R. W. Liu, V. C. Soon, and Y. F. Huang, "Indeterminacy and identifiability of blind identification," IEEE Trans. Circuits and Systems, vol. 38, no. 5, pp. 499-509, 1991.

[6] Y. Inouye and R. W. Liu, "A system-theoretic foundation for blind equalization of an FIR MIMO channel system," IEEE Trans. Circuits Syst. I, vol. 49, no. 4, pp. 425-436, 2002.

[7] A. Medles and D. T. M. Slock, "Semiblind channel estimation for MIMO spatial multiplexing systems," in Proc. VTC2001-Fall, Oct. 2001, vol. 2, pp. 1240-1244.

[8] C. Cozzo and B. L. Hughes, "Joint channel estimation and data detection in space-time communications," IEEE Trans. Commun., vol. 51, no. 8, pp. 1266-1270, 2003.

[9] S. Buzzi, M. Lops, and S. Sardellitti, "Performance of iterative data detection and channel estimation for single-antenna and multiple-antennas wireless communications," IEEE Trans. Veh. Technol., vol. 53, no. 4, pp. 1085-1104, 2004.

[10] T. Wo, P. A. Hoeher, A. Scherb, and K. D. Kammeyer, "Performance analysis of maximum-likelihood semiblind estimation of MIMO channels," in Proc. VTC2006-Spring, Melbourne, Australia, May 2006, vol. 4, pp. $1738-1742$.
[11] A. K. Jagannatham and B. D. Rao, "Whitening-rotation-based semiblind MIMO channel estimation," IEEE Trans. Signal Processing, vol. 54, no. 3, pp. 861-869, 2006.

[12] M. Abuthinien, S. Chen, A. Wolfgang, and L. Hanzo, "Joint maximum likelihood channel estimation and data detection for MIMO systems,' in Proc. ICC 2007, Glasgow, Scotland, UK, June 2007.

[13] M. Biguesh and A. B. Gershman, "Training-based MIMO channel estimation: a study of estimator tradeoffs and optimal training signals," IEEE Trans. Signal Processing, vol. 54, no. 3, pp. 884-893, 2006.

[14] L. Hanzo, S. X. Ng, T. Keller, and W. Webb, Quadrature Amplitude Modulation: From Basics to Adaptive Trellis-Coded, Turbo-Equalised and Space-Time Coded OFDM, CDMA and MC-CDMA Systems, 2nd edition. Chichester, UK: John Wiley, 2004.

[15] S. Chen and E. S. Chng, "Concurrent constant modulus algorithm and soft decision directed scheme for fractionally-spaced blind equalization," in Proc. ICC 2004, Paris, France, June 2004, vol. 4, pp. 2342-2346.

[16] S. Chen, A. Wolfgang, and L. Hanzo, "Constant modulus algorithm aided soft decision directed scheme for blind space-time equalisation of SIMO channels," Signal Processing, vol. 87, no. 11, pp. 2587-2599, 2007.

[17] Z. Ding, T. Ratnarajah, and C. F. N. Cowan, "HOS-based semi-blind spatial equalization for MIMO Rayleigh fading channels," IEEE Trans. Signal Processing, vol. 56, no. 1, pp. 248-255, 2008.

[18] L. Hanzo, M. Münster, B. J. Choi, and T. Keller, OFDM and MC-CDMA for Broadband Multi-User Communications, WLANs and Broadcasting. Chichester, UK: John Wiley, 2003.

[19] C. B. Papadias, "Unsupervised receiver processing techniques for linear space-time equalization of wideband multiple input/multiple output channels," IEEE Trans. Signal Processing, vol. 52, no. 2, pp. 472-482, 2004.

[20] J. Shen and Z. Ding, "Zero-forcing blind equalization based on subspace estimation for multiuser systems," IEEE Trans. Commun., vol. 53, no. 2, pp. 262-271, 2001.

[21] H.-F. Chen, X.-R. Cao, H.-T. Fang, and J. Zhu, "Nonlinear adaptive blind whitening for MIMO channels," IEEE Trans. Signal Processing, vol. 53, no. 8, pp. 2635-2647, 2005.

[22] D. Godard, "Self-recovering equalization and carrier tracking in twodimensional data communication systems," IEEE Trans. Commun., vol. COM-28, pp. 1867-1875, 1980.

[23] J. R. Treichler and B. G. Agee, "A new approach to multipath correction of constant modulus signals," IEEE Trans. Acoustics, Speech and Signal Processing, vol. ASSP-31, no. 2, pp. 459-472, 1983.

[24] S. Chen, S. McLaughlin, P. M. Grant, and B. Mulgrew, "Multi-stage blind clustering equaliser," IEEE Trans. Commun., vol. 43, no. 3, pp. 701-705, 1995.

[25] S. Chen, H.-Q. Du, and L. Hanzo, "Adaptive minimum symbol error rate beamforming assisted receiver for quadrature amplitude modulation systems," in Proc. VTC2006-Spring, Melbourne, Australia, May 2006, vol. 5, pp. 2236-2240. 